Revista Ibero-Americana de Ciências Ambientais

Ibero-American Journal of Environmental Science

Anais do Simpósio Interdisciplinar de Práticas de Produção

Agrícola e Conservação Ambiental - Jan 2017 - v.8 - n.1

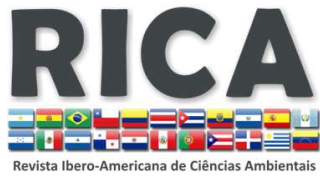

ISSN: 2179-6858

\title{
Diversidade de nematoides em agroecossistema do algodão no Mato Grosso
}

A cultura do algodão tem grande importância econômica e social para o Brasil, no entanto proporciona também impactos ambientais relevantes. Para mensurar esses impactos faz-se uso da dinâmica populacional de nematoides em agroecossistemas como indicador de qualidade de solos. Diante disso, o objetivo do trabalho foi caracterizar a comunidade de nematoides em habitat cultivado com algodão em diferentes estádios fenológicos da cultura. Foram coletadas duas amostras de solo, na profundidade de $30 \mathrm{~cm}$, por área (talhão) e estádio fenológico (inicial , antes da cultura ser implantada; vegetativo; reprodutivo e maturidade fisiológica). Foram realizadas as análises de riqueza, abundância, frequência de ocorrência (FO), índice pontual de abundancia (IPA), Aphelenchus $(9,32 \%)$. Em todas as amostras, o número de indivíduos fitoparasitas foi maior que o de micófagos. Os estádios vegetativo e de maturação fisiológica, apresentaram a maio população de micófagos no talhão 5 (Th5) e no estádio inicial e na maturação fisiológica no talhão 11 (Th11). Quanto à riqueza, os dois talhões apresentaram o mesmo número de gêneros. Sendo identificado como exclusivos o gênero Meloidogyne no Th5 e o gênero Tylenchus no Th11. Considerando a FO, os gêneros Aphelenchus, Helicotylenchus e Pratylenchus foram observados em $75 \%$ das amostras no Th5 e Helicotylenchus (100\%) e Pratylenchus (50\%) no Th11. Resultados semelhantes da FO foram observados para o IPA, acrescentando-se o gênero Aphelenchoides no Th11. Quanto ao $\mathrm{H}^{\prime}$, observou-se uma diversidade total de 0,60 com o valor do Th5 inferior ao Th11. Ao analisar a correspondência canônica (CCA), observou-se que os dados meteorológicos não interferiram diretamente na flutuação populacional de nematoides nos dois talhões. Sendo assim, observou-se que a comunidade de nematoides estudada neste trabalho, foi composta na maior parte, pela população de fitoparasitas e a maturidade fisiológica do algodoeiro foi o estádio fenológico com a maior obtenção de indivíduos tanto fitoparasitas quanto micófagos.

Palavras-chave: Cotonicultura; Nematofauna; Índices Ecológicos.

\section{Nematodes diversity in agroecosystem of cotton in Mato Grosso}

The cotton crop has great economic and social importance to Brazil, however provides significant environmental impacts. Among these environmental impacts, it can mention the population dynamics of nematodes in agroecosystems as an indicator of soil quality. Thus, the aim of this paper was to characterize the nematode community in habitat cultivated with cotton in different phenologicals stages of the crop. It were collected two soil samples at a depth of $30 \mathrm{~cm}$, by area (field) and phenological stage (initial, before the culture is implanted; vegetative; reproductive and physiological maturity). It were realized analyses of richness, abundance, frequency of occurrence (FO), punctual abundance index (PAI), Shannon diversity index ( $\mathrm{H}^{\prime}$ ) it were performed the weather data recorded. At total of 4,400 individuals were identified (abundance), distributed in six families. (Aphelenchidae, Aphelenchoididae, Heteroderidae, Hoplolaimidae, Pratylenchidae e Tylenchidae). The predominant genera were Helicotylenchus (64.77\%), Pratylenchus (15.45\%) and Aphelenchus (9.32\%). In all samples, the number of plant parasites was larger than the mycophagous. The vegetative and physiological maturity stages, had the largest population of mycophagous in field 5 (Th5) and at the initial stage and physiological maturity in field 11 (Th11). As for richness, the two plots had the same number of genus. It was identified as exclusive the genus Meloidogyne in Th5 and Tylenchus in Th11. Considering the FO, the Aphelenchus, Helicotylenchus and Pratylenchus genus were observed in $75 \%$ of samples at the Th5 and Helicotylenchus (100\%) and Pratylenchus (50\%) at the Th11. Similar results of the PO were observed to PAl, adding the genus Aphelenchoides in Th11. As to $\mathrm{H}^{\prime}$, it observed a total range of 0.60 with value in Th5 less than Th11. By analyzing the canonical correspondence (CCA), it observed that meteorological data did not interfere directly in the fluctuation of nematodes in both fields. Thus, it was observed that the nematode community, in this paper, was composed mostly by the population of plant parasites and physiological maturity of the cotton plant was the phenological stage with the greatest achievement of individuals both plant parasites as mycophagous.

Keywords: Cotton Farming, Nematofaune, Ecological Indexes.

Topic: Química Agrícola e Ambiental

Reviewed anonymously in the process of blind peer

\section{Francieli Dominiki Zavislak}

Universidade do Estado de Mato Grosso, Brasil http://lattes.cnpq.br/1093541691522879

franzavislak bio@hotmail.com

Dejânia Vieira Araújo

Universidade do Estado de Mato Grosso, Brasil http://lattes.cnpq.br/3826330763207301

dejania@unemat.br

\section{Vanderlei Antunes Maciel}

Universidade do Estado de Mato Grosso, Brasil

http://lattes.cnpq.br/5145783323744857

vanderkiko@hotmail.com
Received: 20/04/2016

Approved: 24/05/2016

Kethelin Cristine Laurindo de Oliveira

Universidade do Estado de Mato Grosso, Brasi

http://lattes.cnpq.br/1536625767991476

kethelinlaurindo@hotmail.com
Referencing this:

ZAVISLAK, F. D.; ARAÚJO, D. V.; MACIEL, V. A.; OLIVEIRA, K. C. L.. Diversidade de nematoides em agroecossistema do algodão no Mato Grosso. Revista Ibero-Americana de Ciências Ambientais, v.8, n.1, p.129-139, 2017. DOI: http://doi.org/10.6008/SPC21796858.2017.001.0011 


\section{INTRODUÇÃO}

O algodão (Gossypium hirsutum L.) é uma das culturas de maior importância para a produção de fibras mundial, sendo que mais de 60 países são responsáveis pela sua produção, dentre os quais o Brasil ocupa a quinta posição como maior produtor (ABRAPA, 2016). A evolução de seu cultivo atingiu nível tecnológico considerável, tomando como base uma área plantada no país para safra 2014/2015 definida em 976,2 mil hectares, obtendo na região Centro-oeste do Brasil, a produtividade de $1.601 \mathrm{~kg}$ hectare-1 de pluma de algodão (CONAB, 2016).

Paralelamente à expansão da cotonicultura no Brasil, houve também um aumento dos problemas fitossanitários relativos a esta cultura, dentre eles os fitoparasitas. Seu grande potencial em causar prejuízos e comprometer o solo onde o algodão é cultivado fez com que ocupassem posição de destaque entre os vários patógenos que reduzem a produção dessa cultura.

O problema com nematoides patogênicos na cultura do algodão vem sendo estudado em todo o país, contudo não se tem dados consistentes a respeito da caracterização populacional dos principais gêneros, espécies e raças que comumente estão associados a esta cultura, em função do manejo e do sistema de cultivo adotado (FIGUEIRA et al., 2011).

Uma vez introduzidos na área torna-se quase impossível a eliminação dos nematoides presentes nos solos infestados existindo ainda, as particularidades de cada gênero e espécies que devem ser manejadas diferencialmente em cada cultura (TIHOHOD, 2000; FERRAZ, 2010). Com isso, é importante conhecer a biologia e reprodução, forma de dispersão, sobrevivência, hospedeiros e possibilidades de manejo de cada uma delas e assim melhorar a relação de convivência (TOMAZINI et al., 2008).

As comunidades de nematoides possuem diversas formas de adaptação às alterações ambientais ocasionadas por fatores como estresse climático, época de plantio, manejo das culturas, melhoramento genético e fisiologia das plantas. Essa capacidade de adaptação e sobrevivência dos nematoides é importante para entendimento de epidemias e dispersão desses invertebrados sob condições de adversidade vegetal e ambiental (BLAKELY et al., 2002; RITZINGER et al., 2010).

Assim, devido às especificidades no ciclo de vida, capacidade de persistência no solo e taxa de reprodução entre os grupos, a nematofauna está sendo bastante estudada, empregando-se dados relativos das estruturas tróficas e taxionômicas como indicadores biológicos para mensurar as modificações resultantes do emprego de práticas de manejo próprias dos sistemas agrícolas (BONGERS, 1990; FRECKMAN et al., 1993).

Para conhecer a dinâmica populacional desses microrganismos, faz-se uso de índices ecológicos específicos com o intuito de caracterizar a comunidade por meio do número de indivíduos ou mesmo da quantidade de gêneros.

Os índices visam ainda, investigar o uso da biodiversidade edáfica, funcionando como indicadores biológicos dos agroecossistemas, pois identificam os efeitos do manejo sobre a biodiversidade, além de serem usados para a definição de estratégias de manejo mais sustentáveis e constituir uma base de dados 
biológica para futuros estudos sobre impactos agrícolas nas propriedades. Esses estudos garantem a ciclagem de nutrientes e a dinâmica da matéria orgânica no solo (GOULART et al., 2009). Com isso, o objetivo do trabalho foi caracterizar a nematofauna em áreas de cultivo de algodão.

\section{METODOLOGIA}

O trabalho de pesquisa foi realizado em uma propriedade localizada à margem da rodovia MT 364, km 328, município de Diamantino - MT, sob as coordenadas 144'47'S e 5727'18' W. A propriedade possui uma área plantada de 34.257 hectares dividida em 100 talhões com tamanho médio de 300 hectares, onde se cultiva: algodão, milho (Zea mays L.), girassol (Helianthus annuus L.), soja (Glycine max L.) e milheto (Pennisetum glaucum L.).

Foram selecionados dois talhões para realização da pesquisa, sendo o talhão 5 (Th5) com área de 179,07 hectares, onde foi implantado o algodão sem revolvimento do solo, utilizando os restos culturais da soja. O outro talhão selecionado foi o 11 (Th11) com 300,65 hectares, no qual o algodão foi implantado sob a palhada de milheto (com revolvimento do solo). Foram usadas diferentes coberturas de solo para comparar o desenvolvimento das populações de nematoides. Os talhões foram semeados com a cultivar FM 975 WS.

As amostras de solo e de plantas da cultura de algodão foram coletadas na safra 2012/2013, correspondendo ao período de dezembro de 2012 a julho de 2013. Foram realizadas quatro coletas: a primeira no período inicial da cultura, com a cultura anterior ainda implantada, a segunda no período vegetativo (Th5 e Th11 $-V_{3}$ ), terceira no período reprodutivo (Th5 $-F_{3}$; Th11 $-F_{1}$ ) e quarta no período de maturidade fisiológica do algodão (Th5 e Th11 - $C_{5}$ ).

As coletas foram adaptadas de Tomazini et al. (2008), sendo formadas por duas amostras compostas, por área e estádio fenológico, coletadas até a profundidade de $30 \mathrm{~cm}$, com volume de cerca de um litro de solo, cada uma formada por dez subamostras simples que eram demarcadas com auxílio de GPS da marca $\operatorname{Garmin}^{\mathrm{TM}}$, modelo eTrex H. Para demarcação dos pontos de coleta foram desprezados $100 \mathrm{~m}$ de bordadura e demarcadas cinco áreas dentro do talhão, sendo realizadas quatro amostras por área. As coletas de plantas foram realizadas nos mesmos pontos das coletas de solo de forma intercaladas, onde se utilizou duas amostras compostas, cada uma formada por cinco subamostras simples. As plantas coletadas foram armazenadas juntamente com as amostras de solo.

As amostras de solo e plantas foram primeiramente mantidas em recipientes plásticos e depois transferidas para sacos plásticos devidamente identificados e conservados em isopor com gelo. Ao término da coleta, as amostras foram encaminhadas ao laboratório especializado da Associação dos Produtores de Sementes de Mato Grosso - APROSMAT, situado na Rua das Andradas 688, Vila Goulart Rondonópolis - MT para identificação de gênero de nematoides.

Os dados meteorológicos de irradiação, temperatura, umidade relativa do ar e pluviosidade foram obtidos de estação meteorológica da Universidade do Estado de Mato Grosso, campus de Tangará da Serra situada na fazenda vizinha à propriedade. Os dados foram coletados durante todo o período de execução das 
coletas a campo. Estes dados foram correlacionados com a diversidade dos diferentes gêneros de nematoides encontrados.

O número de indivíduos usados para as análises foi obtido pela soma dos encontrados no solo e nas raízes. Os dados foram analisados utilizando-se a estatística não-paramétrica, para as análises de riqueza (NUNES, 2010), abundância absoluta e relativa (NUNES, 2010), frequência de ocorrência (FO) (VIELLIARD et al., 1990), índice pontual de abundância (IPA) (VIELLIARD et al., 1990), índice de diversidade de Shannon (H') (SHANNON et al., 1949) e análise de correspondência canônica (CCA) (HOTELLING, 1935; HOTELLING, 1936) para os dados meteorológicos. Os índices foram obtidos utilizando o programa DIVES (RODRIGUES, 2007) e R (FILHO et al., 2006).

\section{RESULTADOS}

Foram encontrados um total de 4.400 indivíduos, distribuídos em seis famílias: Aphelenchidae, Aphelenchoididae, Heteroderidae, Hoplolaimidae, Pratylenchidae e Tylenchidae. Em relação ao número de indivíduos coletados (abundância), Helicotylenchus spp. foi o mais representativo compreendendo 2.850, ou seja, 64,77\% dos indivíduos (Tabela 1), seguido do gênero Pratylenchus com 15,45\% (680 indivíduos) e do gênero Aphelenchus com 9,32\% (410 indivíduos).

O Th5 apresentou maior número de indivíduos (3.090) se comparado ao Th11 (1.310) (Tabela 1). Vale ressaltar que o algodão no Th5 foi implantado sobre restos culturais de soja, sendo um dos prováveis motivos pelo qual apresentou o maior número de indivíduos, tanto fitoparasitas quanto micófagos. A abundância absoluta de nematoides parasitas de plantas (3.650) foi maior que o número de micófagos (750), tendo prevalecido os indivíduos do gênero Helicotylenchus (Tabela 1).

Tabela 1: Abundância da nematofauna das áreas amostradas na safra 2012/2013. Gênero, abundância absoluta, abundância absoluta total, abundância relativa por gênero e nível trófico.

\begin{tabular}{|c|c|c|c|c|}
\hline \multirow{2}{*}{ Gêneros } & \multicolumn{2}{|c|}{ Abundância absoluta* } & \multirow[b]{2}{*}{ Abundância absoluta total } & \multirow[b]{2}{*}{ Abundância relativa total (\%) } \\
\hline & Th5 & Th11 & & \\
\hline & & & Parasitas de plantas & \\
\hline Helicotylenchus & 2270 & 580 & 2850 & 64,77 \\
\hline Meloidogyne & 120 & 0 & 120 & 2,73 \\
\hline Pratylenchus & 400 & 280 & 680 & 15,45 \\
\hline \multirow[t]{2}{*}{ Subtotal } & 2790 & 860 & 3650 & 82,95 \\
\hline & \multicolumn{4}{|c|}{ Micófagos } \\
\hline Aphelenchoides & 90 & 240 & 330 & 7,50 \\
\hline Aphelenchus & 210 & 200 & 410 & 9,32 \\
\hline Tylenchus & 0 & 10 & 10 & 0,23 \\
\hline Subtotal & 300 & 450 & 750 & 17,05 \\
\hline Total & 3090 & 1310 & 4400 & 100,00 \\
\hline
\end{tabular}

* Th5: talhão 5; Th11: talhão 11.

Com relação à riqueza de espécies de nematoides os dois talhões apresentaram o mesmo número de gêneros. 0 Th5 apresentou apenas um gênero exclusivo, Meloidogyne, e o Th11 também apresentou um gênero exclusivo, Tylenchus (Tabela 1). A exclusividade de Meloidogyne pode estar associada ao não 
revolvimento do solo, uma vez que esta prática não expõe os ovos a alta temperatura, favorecendo o desenvolvimento dos indivíduos.

No Th5, o número de indivíduos fitoparasitas $(90,29 \%)$ foi maior que o número de micófagos $(9,71 \%)$. O mesmo resultado foi observado no Th11, onde o número de fitoparasitas e micófagos chegou a 65,66\% e $34,35 \%$ respectivamente (Figura 1 ).

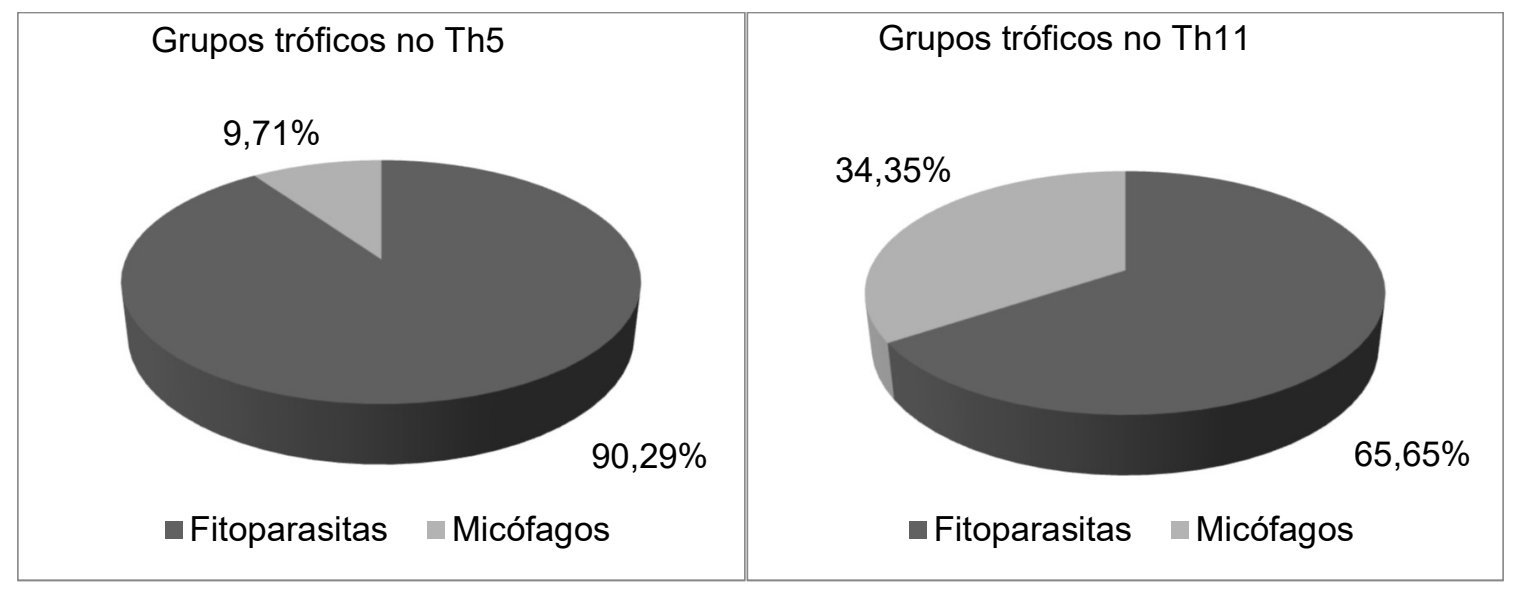

Figura 1: Grupos tróficos de nematoides em habitats cultivados com algodão. As médias são referentes às quatro épocas de coleta. Th5 - talhão 5; Th11 - talhão 11.

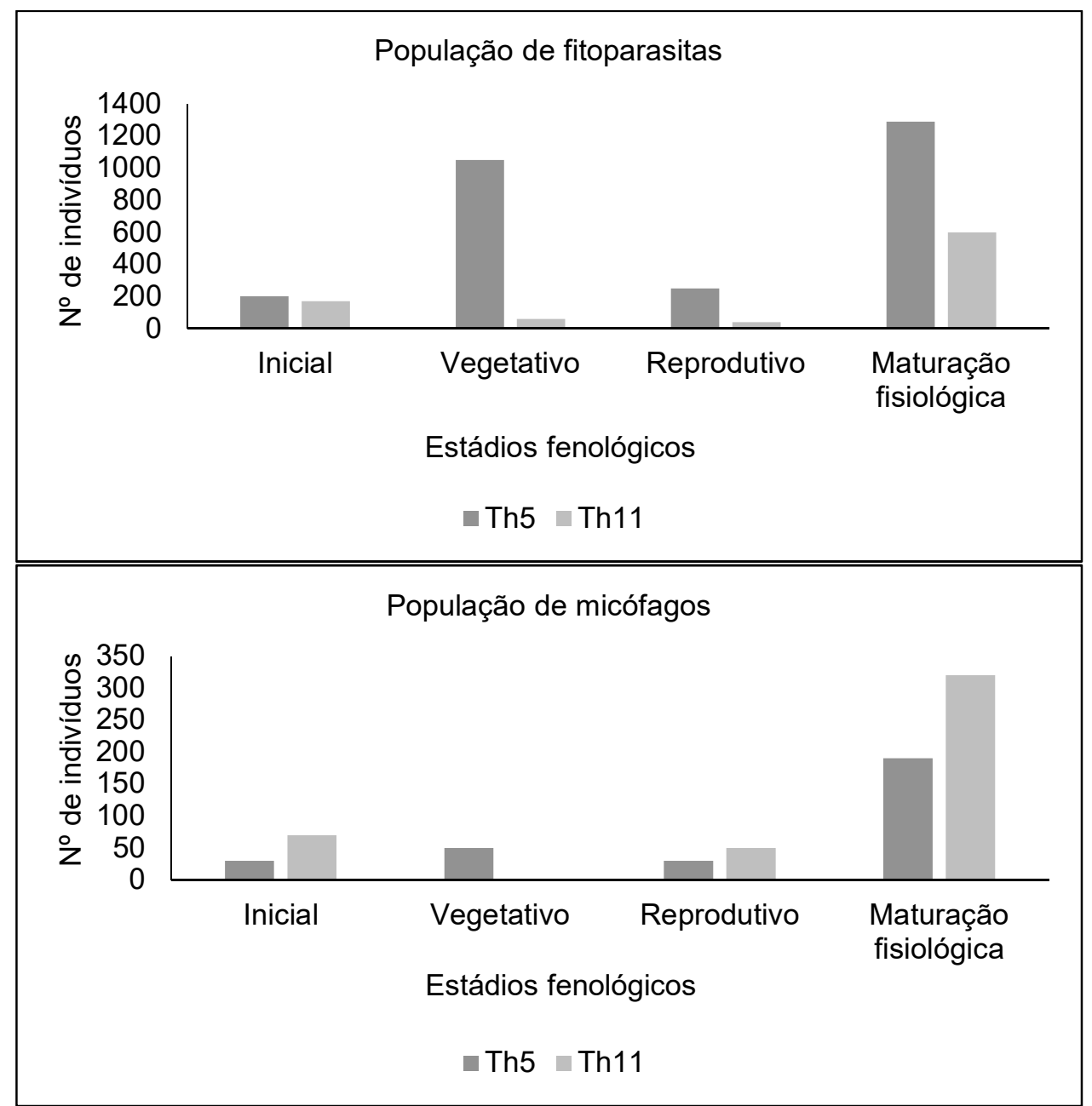

Figura 2: População de fitoparasitas e micófagos da cultura do algodão nas quatro épocas de coleta: inicial, vegetativo, reprodutivo e maturação fisiológica. Th5 - talhão 5 e Th11 - talhão 11. 
No Th5, o estádio vegetativo e de maturação fisiológica foram os que apresentaram o maior número de indivíduos fitoparasitas. No Th11 os estádios, inicial e de maturação fisiológica, foram os mais expressivos para estes indivíduos. Resultados semelhantes a estes foram observados na população de micófagos, nos dois talhões estudados, não sendo registrado nenhum indivíduo no vegetativo do Th11 (Figura 2).

Considerando a frequência de ocorrência (FO), destacaram-se no Th5 os gêneros: Aphelenchus, Helicotylenchus e Pratylenchus presentes em 75\% das coletas. Semelhantemente no Th11, destacaram-se os mesmos gêneros, Helicotylenchus (100\%), Aphelenchus e Pratylenchus, ambos com 50\% de FO. A exceção para Aphelenchoides que neste talhão foi observado 62,5\% de FO (Tabela 2).

No índice pontual de abundância (IPA) registrou 386,25 contatos no Th5 e 163,75 contatos no Th11. Destacando-se no Th5 os gêneros: Helicotylenchus (283,75 contatos), Pratylenchus (50 contatos) e Aphelenchus (26,25 contatos). No Th11 destacaram-se os gêneros: Helicotylenchus ( 72,5 contatos), Pratylenchus (35 contatos) e Aphelenchoides (30 contatos) (Tabela 2).

Tabela 2: Nematofauna com indicação do hábitat, frequência de ocorrência (FO) e índice pontual de abundância (IPA) para a cultura do algodão.

\begin{tabular}{lccccc}
\hline \multirow{2}{*}{ Gêneros } & \multirow{2}{*}{ Hábitat** } & \multicolumn{2}{c}{ FO (\%)* } & \multicolumn{2}{c}{ IPA* } \\
\cline { 3 - 6 } & & Th5 & Th11 & Th5 & Th11 \\
\hline Aphelenchoides & 5,11 & 12,50 & 62,50 & 11,25 & 30,00 \\
Aphelenchus & 5,11 & 75,00 & 50,00 & 26,25 & 25,00 \\
Helicotylenchus & 5,11 & 75,00 & 100,00 & 283,75 & 72,50 \\
Meloidogyne & 5 & 12,50 & 0,00 & 15,00 & 0,00 \\
Pratylenchus & 5,11 & 75,00 & 50,00 & 50,00 & 35,00 \\
Tylenchus & 11 & 0,00 & 12,50 & 0,00 & 1,25 \\
\hline Total & & & & 386,25 & 163,75 \\
\hline
\end{tabular}

* Áreas de coleta: Th5 - talhão 5; Th11 - talhão 11; ** 5: talhão 5; 11: talhão 11.

$\mathrm{Na}$ Tabela 3 estão apresentados os valores de diversidade de Shannon (pelo qual se atribuem pesos iguais aos táxons independentemente de suas abundâncias) para os dois talhões. O Th5 apresentou menor $H^{\prime}(0,47)$ do que o Th11 $(0,65)$, sendo observado um $H^{\prime}$ de 0,60 .

Tabela 3: Valores do Índice de diversidade de Shannon $\left(\mathrm{H}^{\prime}\right)$ por talhão.

\begin{tabular}{ll}
\hline Área* & $\mathrm{H}^{\prime}$ \\
\hline Th5 & 0,47 \\
Th11 & 0,65 \\
Total* & 0,60 \\
\hline
\end{tabular}

* Áreas de coleta: Th5 - talhão 5; Th11 - talhão 11.

Com relação aos estádios fenológicos estudados, os valores de $\mathrm{H}^{\prime}$ variaram dentro de cada talhão, sendo que no Th5 a maior diversidade foi encontrada no estádio inicial da cultura $(0,47)$, diferentemente do Th11 que apresentou maior diversidade no estádio de maturação fisiológica $(0,57)$ (Tabela 4).

Tabela 4: Valores do Índice de diversidade de Shannon $\left(\mathrm{H}^{\prime}\right)$ por talhão e estádio fenológico.

\begin{tabular}{llc}
\hline Área* & Estádio fenológico & $\mathrm{H}^{\prime}$ \\
\hline Th5 & Inicial & 0,47 \\
Th5 & Vegetativo & 0,31 \\
Th5 & Reprodutivo & 0,15 \\
Th5 & Maturação fisiológica & 0,28 \\
Th11 & Inicial & 0,37
\end{tabular}


Th11

Vegetativo

* Áreas de coleta: Th5 - talhão 5; Th11 - talhão 11.

O experimento foi realizado no município de Diamantino a $14^{\circ} 5^{\prime} 01^{\prime \prime}$ de latitude Sul e $56^{\circ} 29^{\prime} 37^{\prime \prime}$ longitude Oeste, com altitude média de $463 \mathrm{~m}$, temperatura variando de 15 a $35^{\circ} \mathrm{C}$ durante o ano, a precipitação da região varia de $270 \mathrm{~mm}$ na época das águas e de $20 \mathrm{~mm}$ na época da seca. 0 solo da área é classificado como Latossolo Vermelho (LV) de textura argilosa (FERREIRA, 2001).

A análise dos dados meteorológicos e a abundância dos gêneros por meio da análise de correspondência canônica (CCA) permitiu a divisão deles em três grupos, o primeiro formado por temperatura e irradiação, o segundo por pluviosidade e o terceiro por umidade (Figura 3).

Percebeu-se a interferência de temperatura e irradiação para espécie 1 (sp1) e 6 (sp6), de pluviosidade para espécie 3 (sp3) e de umidade para espécie 4 (sp4), mas essa interação não foi tão íntima, deixando as espécies afastadas do eixo com as características meteorológicas. Isso não permitiu afirmar que foram essas características que interferiram diretamente na flutuação populacional desses gêneros. As espécies 2 (sp2) e 5 (sp5) ficaram totalmente fora do plano de alcance desses dados (Figura 3).

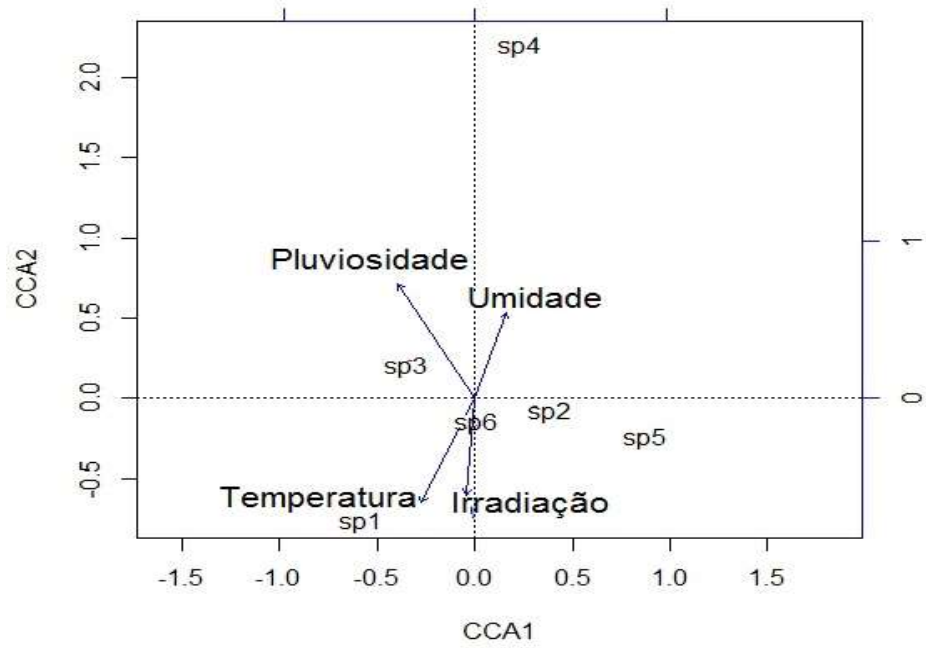

Figura 3: Análise de correspondência canônica para as variáveis meteorológicas: irradiação, pluviosidade, temperatura e umidade para a cultura do algodoeiro. Sp1: Aphelenchoides spp.; sp2: Aphelenchus spp.; sp3: Helicotylenchus spp.; sp4: Meloidogyne spp.; sp5: Pratylenchus spp.; sp6: Tylenchus spp.

\section{DISCUSSÃO}

Assim como no presente trabalho, altas populações do gênero Helicotylenchus também foram encontradas por Arieira (2012), bem como por Tomazini et al. (2008) e Rodrigues et al. (2011) ao estudarem a diversidade de nematoides em sistemas de culturas e manejo do solo.

Overstreet et al. (1996) também evidenciaram em suas pesquisas níveis altos de Helicotylenchus spp. (68\%), o mesmo ocorreu com Gazaway et al. (2003) que encontraram porcentagens de até $81 \%$. Além disso, os autores destacam que mesmo este patógeno sendo considerado pouco conhecido, tem sido prevalente nas áreas estudadas. Isso requer maior atenção do produtor com relação a este gênero, devido às plantas 
apresentarem sintomas semelhantes (mau desenvolvimento das raízes) aos causados por Pratylenchus, sendo facilmente confundidos.

Asmus (2004) ao estudar a população de nematoides em diferentes municípios do Mato Grosso do Sul, encontrou índices populacionais semelhantes para Pratylenchus, não superando 220 nematoides por $200 \mathrm{~cm}^{3}$ de solo.

A associação de indivíduos do gênero Meloidogyne com outros nematoides de importância econômica em lavouras de algodão foi relatada por vários autores. Asmus (2004), em levantamento realizado no Mato Grosso do Sul com a cultura do algodoeiro, relatou a presença de $M$. incognita em $27,7 \%$ das áreas amostradas e $P$. brachyurus em $65,2 \%$, o autor associou o fato ao tipo de solo encontrado nas plantações, sendo que o primeiro possui maior afinidade com solos arenosos, enquanto o segundo é mais encontrado em solos argilosos.

Várias espécies de nematoides parasitam o algodoeiro, porém, Meloidogyne incognita é frequentemente associado às grandes perdas na produção dessa cultura, o que requer maior atenção quanto ao manejo desse fitoparasita (FREITAS et al., 1999).

No estado do Paraná, Silva et al. (1994) também verificaram a presença de $M$. incognita em algumas regiões com presença de sintomas severos. Silva et al. (1997), ao realizarem trabalho com algodão em cinco municípios do Triângulo Mineiro - MG encontraram além de $M$. incognita, gêneros como Pratylenchus, Helicotylenchus, Ditylenchus, Aphelenchus, Rodopholus e Criconemella.

A alta população de fitoparasita encontrada neste trabalho se deve principalmente ao elevado número de indivíduos do gênero Helicotylenchus, este nematoide embora seja considerado fraco é frequentemente encontrado em áreas cultivadas. Essa espécie é ectoparasita migrador e, seus prováveis danos, ocorrem em níveis populacionais elevados (BONFIM JÚNIOR, 2013).

O número expressivo de micófagos é um dado positivo, pois essas populações prevalecem nos sistemas com vegetação nativa, ou seja, o impacto ambiental no solo dos talhões não alcançou nível considerado problemático (TOMAZINI et al., 2008).

Nos últimos anos, alguns estudos têm abordado o uso de nematoides entomopatogênicos e/ou suas bactérias simbiontes como antagonistas de nematoides fitoparasitas. Contudo, em geral esses trabalhos não esclarecem os mecanismos envolvidos na interação entre os dois nematoides (JAGDALE et al, 2002; SOMASEKHAR et al., 2002; LEWIS et al., 2005). Mas o decréscimo do número de entomopatogênicos facilita o desenvolvimento dos fitopatogênicos.

Outro fato é a influência do estádio fisiológico da cultura que, neste caso, apresentou maior número de micófagos e fitoparasitas no estádio de maturação fisiológica da planta, provavelmente por essa já ter gastado a maior parte da reserva para desenvolvimento da maçã.

O algodoeiro, cultivado nos dois talhões estudados, apresentou uma baixa FO e IPA de nematoides se comparado a outras culturas como soja e milho (dados não publicados). Em pesquisa comparando culturas usadas em sucessão, observou-se que o algodão reduziu a multiplicação de Pratylenchus sp. em 10\% em 
amostras de solo (INOMOTO, 2011). Uma explicação para tal fato é o uso de Plantio Direto no talhão, pois a rotação de cultura dificulta o desenvolvimento dos nematoides.

Arieira (2012) afirmou que altas diversidades de nematoides foram observadas nas áreas sob sistema de rotação de culturas, em comparação à sucessão soja/trigo. No entanto, em algumas pesquisas foram observados que os índices mensuradores de diversidade das comunidades podem ser pouco eficientes em caracterizar sistemas agrícolas, por não serem tão precisas. Para compensar isso, recomenda-se a utilização dos índices de abundância e da riqueza de gêneros como ferramentas mais indicadas, uma vez que estes seguem o valor real encontrado (MATTOS et al., 2006; TOMAZINI et al., 2008).

Figueira et al. (2011) ao estudarem a estrutura da população de nematoides do solo no estado do Rio de Janeiro afirmaram que o $\mathrm{H}^{\prime}$ indicou que a rotação de culturas e a sazonalidade são fatores que influenciam a variabilidade da nematofauna. Este resultado foi semelhante ao encontrado por García-Álvarez et al. (2004) que analisaram o efeito da atividade agrícola em sistemas orgânico e convencional de cereais.

O maior número de fitoparasitas em Th11 pode ser consequência do plantio na palha realizado sobre a cultura do milheto, uma vez que a sucessão garante o sucesso no desenvolvimento dos indivíduos. A falta de interação entre nematoides e as variáveis climáticas na cultura do algodoeiro também foram encontradas por Asmus et al. (2009) ao analisarem populações de Rotylenchulus reniformis, em uma fazenda em Aral Moreira.

Pode-se inferir que as variáveis ambientais não interferiram significativamente na flutuação populacional dos nematoides encontrados ao longo de período de coletas na presente pesquisa. Assim, o desenvolvimento de estudos de longa duração que busquem conhecer a flutuação populacional da nematofauna do solo, bem como encontrar padrões de mudanças em sua composição, levando-se em conta as variáveis ambientais e alterações do uso da terra, são de grande contribuição para uma melhor compreensão dos efeitos das áreas cultivadas sobre a estrutura do solo.

\section{CONCLUSÕES}

Baseando-se em índices ecológicos, o ambiente cultivado com algodão favoreceu a população de nematoides de fitoparasitas. Apesar de apresentar alta população de nematoides micófagos no Th11 cultivado sob palhada de milheto.

Prevaleceu a ocorrência dos gêneros Pratylenchus, Aphelenchoides, Aphelenchus e Helicotylenchus. Este fato é de suma importância, tendo em vista o aumento da área cultivada com as culturas de soja, milho e algodão, principalmente na região Centro-Oeste, tornando-se necessário o aprofundamento dos estudos desses gêneros, ressaltando que esse último mesmo não considerado como praga chave atingiu altos níveis populacionais nessa pesquisa.

A maturidade fisiológica do algodoeiro foi o estágio fenológico com a maior obtenção de indivíduos fitoparasitas e micófagos, sendo o Th11 com menor população de fitoparasitas. 


\section{AGRADECIMENTOS}

À Coordenação de Aperfeiçoamento de Pessoal de Nível Superior (CAPES) pela concessão de bolsa de Mestrado ao primeiro autor. À Universidade do Estado de Mato Grosso (UNEMAT) e ao Programa de PósGraduação Sensu Stricto em Ambiente e Sistemas de Produção Agrícola (PPGASP).

\section{REFERÊNCIAS}

ABRAPA. Estatísticas: $O$ algodão no Brasil. Brasília: ABRAPA, 2016.

ARIEIRA, G. O.. Diversidade de nematoides em sistemas de culturas e manejo do solo. Dissertação (Mestrado em Agronomia) - Universidade Estadual de Londrina, Londrina, 2012.

ASMUS, G. L.; ISHIMI, C. M.. Flutuação populacional de Rotylenchulus reniformis em solo cultivado com algodoeiro. Pesquisa Agropecuária Brasileira, Brasília, v.44, n.1, p.51-57, 2009.

ASMUS, G. L.. Ocorrência de nematoides fitoparasitos em algodoeiro do estado de Mato Grosso do Sul. Nematologia Brasileira, Cassilândia, v.28, n.1, p.77-86, 2004.

BLAKELY, J. K.; NEHER, D. A.; SPONGBERG, A. L.. Soil invertebrate and microbial communities, and decomposition as indicators of polycyclic aromatic hydrocarbon contamination. Applied Soil Ecology, Amsterdam, v.21, n.1, p.71-88, 2002.

BONFIM JÚNIOR, M. F.. Nematoides em feijoeiro-comum: ocorrência nos Estados do Paraná e São Paulo, e interação de cultivares com Pratylenchus brachyurus, Meloidogyne incognita e Meloidogyne javanica. Tese (Doutorado em Fitopatologia) - Escola Superior de Agricultura Luiz de Queiroz, Piracicaba, 2013.

BONGERS, T.. The maturity index: an ecological measure of nematoides disturbance based on nematodes species composition. Oecologia, Heidelberg, v.83, n.1, p.14-19, 1990.

CONAB. Acompanhamento de safra brasileira: grãos, nono levantamento, fevereiro 2016. Brasília: Conab, 2016.

FERRAZ, S.. Manejo sustentável de fitonematoides. Viçosa: UFV, 2010.

FERREIRA, J. C. V.. Mato Grosso e seus municípios. 19 ed. Cuiabá: Buriti, 2001.

FIGUEIRA, F. F.; BERBARA, R. L. L.; PIMENTEL, J. P.. Estrutura da população de nematoides do solo em uma unidade de produção agroecológica no Estado do Rio de Janeiro, Brasil. Acta Scientiarum Agronomy, Maringá, v.33, n.2, p.223-229, 2011.

FILHO, A.; CAVALCANTE, C.. Enfoque estatístico usando o Software R. Belo Horizonte:

Geocities, 2006. intervention. Agriculture, Ecosystems \& Environment, Amsterdam, v.45, n.2, p.239-261, 1993.

FREITAS, L. G.; OLIVEIRA, R. D. L. O.; FERRAZ, S.. Introdução à nematologia. Viçosa: UFV, 1999.

GARCÍA-ÁLVAREZ, A.; ARIAS, M.; DÍEZ-ROJO, M. A.; BELLO, A.. Effect of agricultural management on soil nematode trophic structure in a Mediterranean cereal system. Applied Soil Ecology, Amsterdam, v.27, n.3, p.197-210, 2004.

GAZAWAY, W. S.; MCLEAN, K. S. A.. Survey of plant-parasitic nematodes associated with cotton in Alabama. Journal of Cotton Science, Baton Rouge, v.7, n.1, p.1-7, 2003.

GOULART, A. M. C.; CARES, J. E.; FERRAZ, L. C. C. B.. Ecologia e biodiversidade de nematoides - parte I. Revisão Anual de Patologia de Plantas, Passo Fundo, v.17, n.1, p.149-188, 2009

HOTELLING, H.. The most predictable criterion. Journal of Educational Psychology, Washington, v.26, n.1, p.139-142, 1935.

HOTELLING, H.. Relations between two sets of variables. Biometrika, Oxford, v.28, n.1, p.321-377, 1936. INOMOTO, M. M. Avaliação da resistência de 12 híbridos de milho a Pratylenchus brachyurus. Tropical Plant Pathology, Brasília, v.36, n.5, p.308-32, 2011.

JAGDALE, G. B.; SOMASEKHAR N.; GREWAL, P. S.; KLEIN, M. G.. Suppression of plant-parasitic nematodes by application of live and dead infective juveniles of an entomopathogenic nematode, Steinernema carpocapsae, on boxwood (Buxus ssp.). Biological Control, Washington, v.24, n.1, p.42-49, 2002.

LEWIS, E. E.; GREWAL, P. S.. Effects of entomopathogenic nematodes on plante-parasitic nematodes. In: GREWAL, P. S.; EHLERS R. U.; SHAPIRO-ILAN, D.. Nematodes as Biocontrol Agents. Wallingford: CABI Publishing, 2005. p.349-361.

MATTOS, J. K. A.; HUANG, S. P.; PIMENTEL, C. M. R. M. Grupos tróficos da comunidade de nematoides do solo em oito sistemas de uso da terra nos cerrados do Brasil central. Nematologia Brasileira, Piracicaba, v.30, n.3, p.267-273, 2006.

NUNES, J. R. S.. Avifauna do Rio Paraguai, Pantanal de Cáceres, Mato Grosso. Tese (Doutorado em Ecologia e Recursos Naturais) - Universidade de São Carlos, São Carlos, 2010. 
OVERSTREET, C.; MCGAWLEY, E. C.. Current incidente of plant parasiti cnematodes in Louisiania. Nashville: Beltwide cotton conferences, 1996.

RITZINGER, C. H. S. P.; FANCELLI, M.; RITZINGER, R. Nematoides: bioindicadores de Sustentabilidade $e$ mudanças edafoclimáticas. Revista Brasileira de Fruticultura, Jaboticabal, v.32, n.4, p.1289-1296, 2010.

RODRIGUES, C. V. M. A.; PEDROSA, E. M. R.; OLIVEIRA, A. K. S.; LEITÃO, D. A. H. S.; BARBOSA, N. M. R.; OLIVEIRA, N. J. V.. Distribuição vertical da nematofauna associada à cana-deaçúcar. Nematropica, Aubum, v.41, n.1, p.5-11, 2011.

RODRIGUES, W. C.. DIVES: Diversidade de Espécies - Guia do Usuário. Seropédica: Entomologistas do Brasil, 2007.

SHANNON, C. E.; WEAVER, W.. The Mathematical Theory of Communication. Urbana: University of Illinois Press, 1949.

SILVA, C. M.; SANTOS, M. A.. Levantamento de nematoides na cultura do algodoeiro. Nematologia Brasileira, Piracicaba, v.21, n.1, p.22-23, 1997.
SILVA, J. F. V.; CARNEIRO, R. G.. Levantamento de nematoides associados a cultura de algodão no Paraná. Nematologia Brasileira, Piracicaba, v.18, n.1-2, p.73-78, 1994.

SOMASEKHAR, N.; GREWAL, P. S.; De NARDO E, A. B. STINNER B. R.. Non-target effects of entomopathogenic nematodes on the soil nematode community. Journal of Applied Ecology, Amsterdam, v.39, n.1, p.735-744, 2002.

TIHOHOD, D.. Nematologia agrícola aplicada. Jaboticabal: FUNEP, 2000

TOMAZINI, M. D.; FERRAZ, L. C. C. B.; MONTEIRO, A. R. Abundância e diversidade de nematoides em áreas contíguas de vegetação natural e submetidas a diferentes tipos de uso agrícola. Nematologia Brasileira, Piracicaba, v.32, n.3, p.185-193, 2008

VIELLIARD, J.; SILVA, W. R.. Nova metodologia de levantamento quantitativo e primeiros resultados no interior do Estado de São Paulo. In: ENCONTRO NACIONAL DE ANILHADORES DE AVES, 4. Anais. Recife: UFRPe, 1990. 